\title{
Preliminary Study OF THE Influence OF ManUfaCtURing PARAMETERS IN FuSED Deposition MODELING
}

\author{
A.P. Valerga, M. Batista, S.R. Fernández-Vidal, A. Gómez-Parra, M. Marcos \\ University of Cádiz, Mechanical Engineering and Industrial Design Dept. Faculty of Engineering. Av. Universidad de \\ Cádiz 10, E-11519, Puerto Real, Cádiz, SPAIN
}

\begin{abstract}
Fused Deposition Model is one of the main Additive Manufacturing processes. Nevertheless it has some limiting features. One of them is the surface quality of the product.

For this technique, a specimen has been proposed through the modification of two important manufacturing parameters: the feed rate and the extrusion temperature. This allows analyzing the impact of such parameters, both individually and jointly, in the quality of the generated surface. The material selected for this specimen has been Polylactic Acid (PLA). The theoretical surface roughness has been determined as a function of the two studied process parameters. The deduced equations can be used to calculate the quality of the manufactured parts.
\end{abstract}

Keywords: FDM; Additive Manufacturing; Roughness; Temperature; Production rate
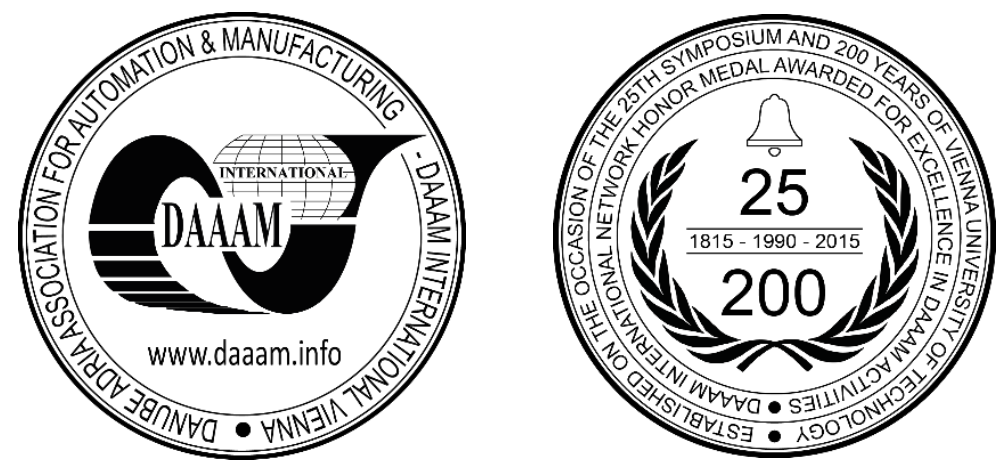

This Publication has to be referred as: Valerga, A[na] P[ilar]; Batista, M[oises]; Fernandez, S[evero] R[aul]; GomezParra, A[lvaro] \& Barcena, M[ariano] (2016). Preliminary Study of the Influence of Manufacturing Parameters in Fused Deposition Modeling, Proceedings of the 26th DAAAM International Symposium, pp.1004-1008, B. Katalinic (Ed.), Published by DAAAM International, ISBN 978-3-902734-07-5, ISSN 1726-9679, Vienna, Austria

DOI: 10.2507/26th.daaam.proceedings.141 


\section{Introduction}

Additive manufacturing techniques (AM) have undergone a major expansion in recent years to meet $\mathrm{AM}$ equipment in very broad areas, including some that are away from purely industrial ones. This means that in many cases these techniques are not studied as it would be desirable, especially when they are used for producing prototypes with well defined requirements $[1,2]$.

These requirements seek to develop products of high quality, at lowest cost, in the shortest time as possible. Notwithstanding, there are some aspects or conditions in Additive Manufacturing that may affect the quality of the manufactured parts [3 - 6]. In this regard, in addition to technology, device and material, it is considered that other conditions can affect the results.

Therefore, it is necessary a study on different manufacturing parameters and their influence on the final characteristics of the parts produced, inasmuch as these directly affect the results [7].

In this case, Fused Deposition Modelling (FDM) has been selected as a widely used and low cost technique. FDM is an established rapid prototyping (RP) technology emerged as a possible product realization from conceptual or digital models aimed to shorten time to market, a strong factor in competitiveness. It produces complex shapes directly from computer data, with little use of resources, without geometry limitation and part-specific tooling [8].

A limiting aspect of FDM is the surface quality of produced parts and their geometric deviations [9, 10]. Notice that scarce published works have studied the impact caused for different parameters in the final quality of the obtained product using this technique. Thus, there is the need for further research concerning this issue, since this technique is not currently intended only for prototyping, but it is used increasingly to obtain final pieces [3]. However, the surface quality or roughness has been studied mainly by the direction of the deposited filaments or position and orientation of the workpiece on the platform [4, 9-13].

In the current work the aim has been to determine the influence of some manufacturing parameters in terms of surface quality. The evaluated or studied parameters are extrusion temperature, being the most important parameter in this technology [7, 14-17]; and the nozzle speed, since it determines the manufacturing rate or productivity. Although this last parameter is considered to be of great importance, it is barely mentioned in this type of process [15].

Definitely, this work aims to predict the surface roughness of Fused Deposition Modelling prototypes depending on the used temperature and speed. The results are unique to the used equipment and material. However, the influence of these parameters on the results is expected to be maintained. However, more investigation is required performing similar studies for other materials and similar machines.

\section{Experimental Procedure}

Manufacturing specimens to study the influence of the above parameters in results are proposed. Since there is no standard specimens until today in these technologies, a simple geometry was chosen: a cube of $30 \mathrm{~mm}$ edge. The flat walls allow to analyze more easily the roughness of these surfaces. This geometry could also facilitate analyze dimensional deviations in future studies.

The production of various cubes is proposed, keeping all manufacturing parameters constant (Table 1 shows the most important ones) except for the above mentioned, the extrusion temperature and the nozzle or manufacturing speed. Thus, the speed intervals recommended by the machine manufacturer (3D Systems) are used, and the minimum extrusion temperature, 5 degrees above the melting point of the selected material. The values of these intervals are shown in Table 2 and the combination of all of them has been used for the manufacturing samples, obtaining 30 test probes. These specimens were printed directly on the surface of the platform, without using glue or adhesive tape. Greater adherence is checked at higher temperatures.

The main limitation found is the impact of environmental temperature during manufacturing. A not closed machine is used, so that the ambient temperature is not constant, heat dissipation varies and the produced parts will be affected as will be mentioned later.

Once parts have been manufactured, a preliminary analysis of the surface quality has been carried out and the typical errors or defects of this technology have been characterized by stereomicroscopy (SOM). Subsequently, with the use of a profilometer, it has been proceeded to obtain 3 roughness measurements per side, regardless of the base resting directly on the building platform. Thus, $12 \mathrm{Ra}$ measurements have been obtained in the laterals faces and other 3 have been obtained in the upper base.

\begin{tabular}{llllllll}
\hline $\begin{array}{l}\text { Machine } \\
\text { model }\end{array}$ & Material & $\begin{array}{l}\text { Room } \\
\text { temperature }\end{array}$ & Density & $\begin{array}{l}\text { Layer } \\
\text { thickness }\end{array}$ & $\begin{array}{l}\text { Nozzle } \\
\text { diameter }\end{array}$ & $\begin{array}{l}\text { Fans } \\
\text { speed }\end{array}$ \\
\hline Cubex & PLA $1.7 \mathrm{~mm}$ & $\approx 298 \mathrm{~K}$ & $16 \%$ & $0.25 \mathrm{~mm}$ & $0,5 \mathrm{~mm}$ & $50 \%$ & $15 \mathrm{~mm} / \mathrm{s}$ \\
\hline
\end{tabular}

Table 1.Constant parameters 


\begin{tabular}{lll}
\hline \hline 26TH DAAAM INTERNATIONAL S YMPOSIUM ON INTELLIGENT MANUFAC \\
\cline { 2 - 3 } & Melting temperature (K) & Nozzle speed (mm/s) \\
\cline { 2 - 3 } & 458 & 20 \\
473 & 22,5 \\
488 & 25 \\
503 & 27,5 \\
518 & 30 \\
533 & - \\
\hline
\end{tabular}

Table 2. Variable parameters: selected values to study

More than necessary measures were done per side in order to reduce some possible system errors. The similarity between these faces have been checked.

Besides the influence of the studied parameters as variables, with the results it should be suggested the optimum production parameters. It will be check whether these parameters match those specified by the machine manufacturer and the chosen material.

\section{Results and discussion}

First of all, it has been analyzed the increase in the manufacturing temperature and speed impact in microgeometrical surface of the specimens, as seen in some images included in Fig. 1.

The footage clearly shows that the increase in temperature causes a minor distinction between deposited filaments generating a more compact or fused surface.

However, the speed does not affect equally in final result. While at low temperature, the speed increase apparently worsens the surface finish obtained, or at least causes the appearance of many defects, at higher temperatures (above than $480 \mathrm{~K}$ ), the surface quality is improved with an increase in speed. This can be explained because increasing temperature causes more fluid filament and the nozzle must move more quickly for depositing no more than the amount required at each point. Another conjecture might be that maintaining the printhead or nozzle at elevated temperatures longer near to the deposited yarn, favors fluidity and therefore the free expansion of the material. This also causes the material degradation.

This behavior has been observed on all sides, although highest speed repercussion has been shown in the upper base than in the lateral ones. This is because the wires are laid next to each other and there is less heat dissipation.

When it has been analyzed the surface quality in terms of $\mathrm{Ra}$, the similarity of results were found in lateral faces as it has been expected. The regression planes defining the Ra value according to the parameters used are shown in Fig. 2.

In this graph, the trend of the measures of roughness in terms of Ra is represented obtained according to the different used speeds and temperatures, resulting functions $R=f(T, S)$. The figure includes the regression planes corresponding to the four side faces and the upper base of the cube.

The equation that defines the 4 regression planes corresponding to the cube laterals approximately coincides with (1). Equation (2) corresponds to the cube upper base.

$$
\begin{aligned}
& R(T, S)=x^{2,83} * y^{-0,39} * 10^{-4,65} \\
& R(T, S)=x^{2,58} * y^{-1,05} * 10^{-3,38}
\end{aligned}
$$

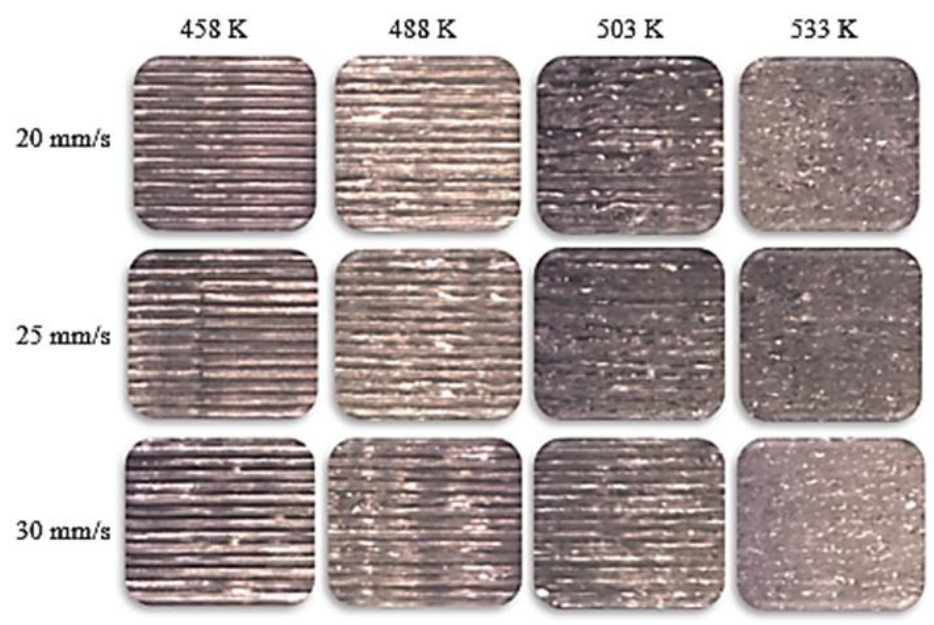

Fig. 1. The increased temperature (left to right) and speed (up and down) impact in the quality apparent surface 


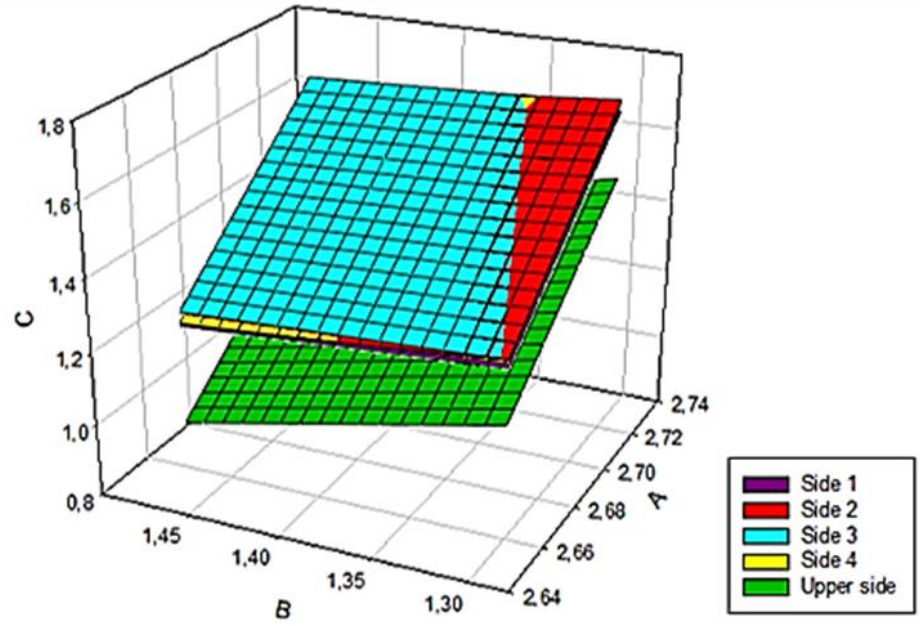

Fig. 2. Plot of Ra trends as function of variable temperature and extrusion speed (log-log planes)

These equations predict a flat surface roughness either parallel or perpendicular to the direction of the head or nozzle displacement or speed. Although they do not predict the surface quality of any orientation, they provide a preliminary idea of the influence of the used parameters in the roughness results. Thus, it appears that for an increase in temperature highest roughness values are obtained, as shown in Fig. 3. Nevertheless, with increased speed, a decrease in $\mathrm{Ra}$ is shown, Fig. 4.

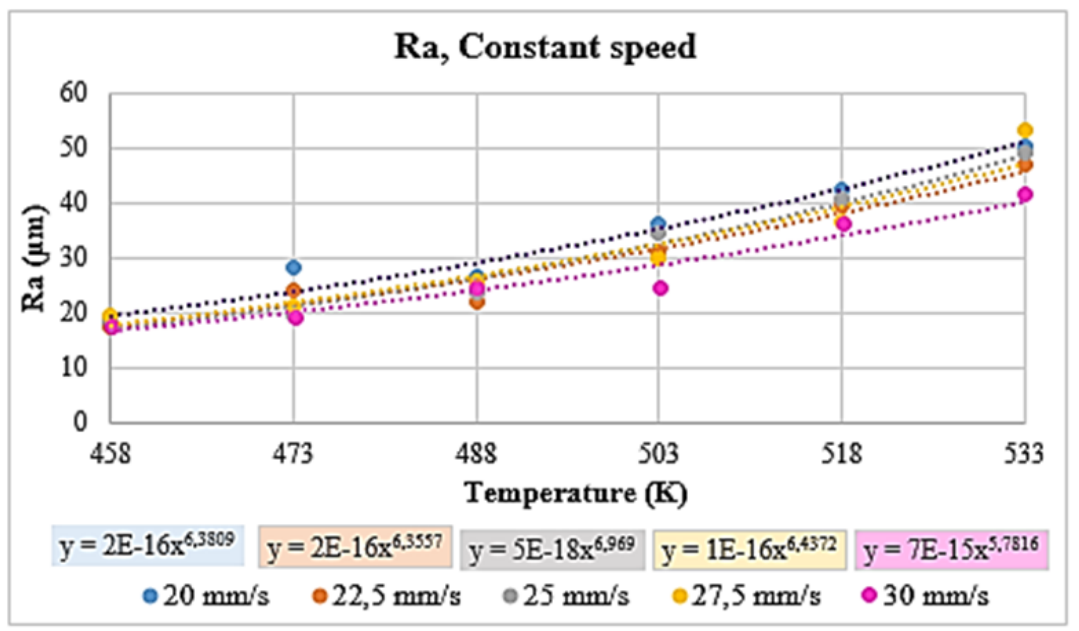

Fig. 3. Ra evolution as the temperature is increased.

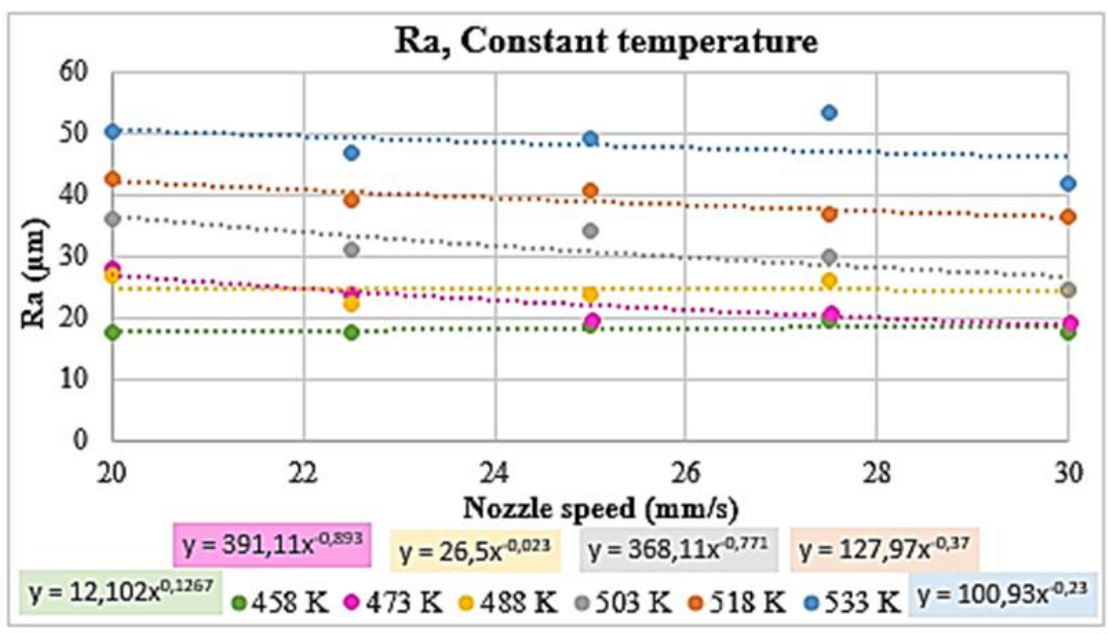

Fig. 4. Ra evolution as nozzle speed is increased 
This means that an increase of temperature causes the worst surface quality, however, an increase in speed can help to improve it. This is otherwise expected.

A hypothesis is set out to stay that less time near the area of material deposition favors heat dissipation and the material is degraded to a lesser extension. This provides better results of Ra. However, there must be a maximum speed above which the accumulation of defects would increase again causing deviations on the surface. Therefore, a proposed future work could be a study of higher amplitude of forward speed range with the possible modification in extrusion speed, which in this case was kept constant. This will may give better roughness results and improve the speed rate and therefore productivity, which is among the most important issues in the industry.

\section{Conclusions}

It have been developed a methodology to study the influence of certain variables in FDM process. With this methodology it has been proposed a model that allows to know the attainable rugosity in advance depending upon these process variables.

Thus, according to the object restrictions, the most appropriate parameters should be selected.

At product development stage, this model is useful to comply with design specifications. Moreover, in process planning it can be applied to increase the manufacturing speed as a good manufacturing strategy. Thus, even better results in surface quality can be obtained.

The predictive model must be checked in other machines and materials, since the process parameters, the machine and the material properties are very different. Therefore, the influence of the parameters in manufactured pieces by FDM is confirmed, but the graphs and equations obtained in other materials and possibly other machines will be not maintained. However, it predicts that trend will be similar.

\section{Acknowledgements}

This work has received financial support from the Andalusian Government (PAIDI) and from the European Union (FEDER/FSE)

\section{References}

[1] S. M. Peltola, F. P. W. Melchels, D. W. Grijpma, A review of rapid prototyping techniques for tissue engineering purposes, Annals of Medicine 40 (4) (2008) 268 - 280.

[2] B. N. Turner, R. Strong, S. A. Gold, A review of melt extrusion additive manufacturing processes: I. Process design and modeling, Rapid Prototyping Journal, 20 (3) (2014) 192 - 204.

[3] H. Singh, F. Rayegani. Cost Optimization of FDM Additive Manufactured parts. Proceedings ASME 2014, Quebec, Canada, 2014.

[4] D. Ahna, J-H. Kweona, S. Kwonb, J. Songb, S. Lee, Representation of surface roughness in fused deposition modeling, Journal of Materials Processing Technology, 209 (2009) 5593 - 5600.

[5] Q. Sun, G. M. Rizvi, C. T. Bellehumeur, Effect of processing conditions on the bonding quality of FDM polymer filaments, Rapid Prototyping Journal, 14 (2) (2008) 72 - 80.

[6] A. Armillotta, Assessment of surface quality on textured FDM prototypes, Rapid Prototyping Journal, 12 (1) (2006) $35-41$.

[7] R. Y. Jiang, Y. H. Gu, Controlling parameters for polymer melting and extrusion in FDM, 12th Grinding and Machining Conference, Kunming, China, 2003.

[8] C. W. Ziemian, P. M. Crawn, Computer aided decision support for fused deposition modeling, Rapid Prototyping Journal, 7 (3) (2001) 138 - 147.

[9] A. Boschetto, V. Giordano, F. Veniali, 3D roughness profile model in fused deposition modelling, Rapid Prototyping Journal, 19/4 (2013) 240 - 252.

[10] A. Cupar, V. Pogačar, Z. Stjepanovič , Shape Verification of Fused Deposition Modelling 3D Prints, International Journal of Information and Computer Science, 4 (2015) 1-8 .

[11] L.M. Galantucci, I. Bodi, J. Kacani, F. Lavecchia, Analysis of Dimensional Performance for a 3D Open-source Printer Based on Fused Deposition Modeling Technique, Procedia CIRP, 28 (2015) 82-8.

[12] J. Zaragoza, H. Medellín, Design for Rapid Prototyping, Manufacturing and tooling: Guidelines. Proceedings ASME 2014, Quebec, Canada, 2014.

[13] R. D. Farahani, K. Chizari, D. Therriault, Three-dimensional printing of freeform helical microstructures: a review, Nanoscale, 6 (2014) 10470 - 10485.

[14] R. B. Dinwiddie, V. Kunc, J. M. Lindal, B. Post, R. J. Smith, L. Love, C. E. Duty, Infrared Imaging of the Polymer 3D-Printing Process, Conference on Thermosense - Thermal Infrared Applications XXXVI, Baltimore, MD, 2014.

[15] A. Boschetto, L. Bottini, Triangular mesh offset aiming to enhance Fused Deposition Modeling accuracy, International Journal of Advanced Manufacturing Technology, 80 (2015) $99-111$.

[16] G. P. Kumar, S. P. Regalla, Optimization of Support Material and Build Time in Fused Deposition Modeling (FDM), Mechanical and Aerospace Engineering (PTS 1-7 Applied Mechanics and Materials), 110 (2012) 2245 - 2251.

[17] P. Jain, A.M. Kuthe, Feasibility Study of Manufacturing Using Rapid Prototyping: FDM Approach, Procedia Engineering, 63 (2013) 4-11. 